\title{
Toll-like receptors are critical for clearance of Brucella and play different roles in development of adaptive immunity following aerosol challenge in mice
}

\author{
Jianwu Pei ${ }^{1,2 *}$, Xicheng Ding ${ }^{1,2}$, Yaping Fan ${ }^{1,2}$, Allison Rice-Ficht ${ }^{1,2,3}$ and Thomas A. Ficht ${ }^{1,2 *}$ \\ 1 Department of Veterinary Pathobiology, Texas A\&M University, College Station, TX, USA \\ 2 Texas AgriLife Research, College Station, TX, USA \\ ${ }^{3}$ Department of Molecular and Cellular Medicine, College of Medicine, Health Science Center, Texas A\&M University, College Station, TX, USA
}

\author{
Edited by: \\ Paul De Figueiredo, Texas A\&M \\ University, USA \\ Reviewed by: \\ Mark Estes, University of Georgia, \\ USA \\ Chad J. Roy, Tulane University, USA \\ *Correspondence: \\ Thomas A. Ficht, Department of \\ Veterinary Pathobiology, Texas \\ A\&M University, College Station, \\ TX 77843-4467, USA \\ e-mail: tficht@cvm.tamu.edu \\ Jianwu Pei, Department of \\ Veterinary Pathobiology, Texas A\&M \\ University, and TX Agrilife \\ Research, College Station, TX, USA. \\ e-mail: jpei@cvm.tamu.edu
}

Brucella spp. cause undulant fever in humans and brucellosis in variety of other animals. Both innate and adaptive immunity have been shown to be important in controlling Brucella infection. Toll-like receptors (TLRs) represent a group of pattern recognition receptors (PRRs) that play critical roles in the host innate immune response, as well as development of adaptive immunity. In the current report, we investigated the role of TLR signaling in the clearance of Brucella and development of adaptive immunity in $\mathrm{TLR2}^{-/-}$, TLR4 ${ }^{-/-}$, or MyD88-/- mice following aerosol exposure to B. melitensis $16 \mathrm{M}$. Consistent with previous reports, MyD88 is required for efficient clearance of Brucella from all three organs (lung, spleen, and liver). The results reveal Th2-skewed immune responses in TLR2 ${ }^{-/}$mice late in infection and support a TLR2 requirement for efficient clearance of Brucella from the lungs, but not from the spleen or liver. Similarly, TLR4 is required for efficient clearance of Brucella from the lung, but exhibits a minor contribution to clearance from the spleen and no demonstrable contribution to clearance from the liver. Lymphocyte proliferation assays suggest that the TLRs are not involved in the development of cell-mediated memory response to Brucella antigen. Antibody detection reveals that TLR2 and 4 are required to generate early antigen-specific IgG, but not during the late stages of infection. TLR2 and 4 are only transiently required for IgM production and not at all for IgA production. In contrast, MyD88 is essential for antigen specific $\lg G$ production late in infection, but is not required for IgM generation over the course of infection. Surprisingly, despite the prominent role for MyD88 in clearance from all tissues, MyD88-knockout mice express significantly higher levels of serum IgA. These results confirm the important role of MyD88 in controlling infection in the spleen while providing evidence of a prominent contribution to protection in other tissues. In addition, although TLR4 and TLR2 contribute little to control of spleen infection, a significant contribution to clearance of lung infection is described.

Keywords: Brucella, TLR, MyD88, aerosol infection, innate immunity, adaptive immunity

\section{INTRODUCTION}

The genus Brucella is a group of Gram-negative, facultative intracellular bacteria that cause brucellosis, a reproductive disease in ruminants, and undulating fever in humans. Brucellosis is one of the most important worldwide zoonotic diseases. Ten species have been identified to date, three of which, including $B$. melitensis, B. abortus, and B. suis are virulent in humans and represent a significant threat to public health (Atluri et al., 2011). Humans often become infected following inhalation of particles carrying the bacteria or consumption of dairy products contaminated with the organism. Although vaccination is used to successfully reduce the spread of disease, the risk remains high in underdeveloped nations. There are currently no vaccines available that are safe for use in humans, and although generally effective, antibiotic treatments do not always prevent disease recrudescence. As a result of these factors and concern over their potential weaponization,
$\mathrm{NIH}$ and the CDC/USDA have classified these three species as category $\mathrm{B}$ agents.

Both innate and adaptive immunity have been described as contributing to the control of Brucella infection (Baldwin and Parent, 2002; Dornand et al., 2002; Baldwin and Goenka, 2006). The role of innate immunity against infection by this pathogen has drawn recent attention as a result of awareness of the role of innate immunity in the establishment of infection and the development of adaptive immunity (Weiss et al., 2005; Oliveira et al., 2011). In contrast, adaptive immunity, including cell-mediated and humoral responses, has been the prominent focus of Brucella research over the past few decades.

The innate immune system is composed of a variety of cellular and humoral components, which are the first line of the host defense against invading pathogens. Recognition relies on pattern recognition receptors (PRRs) expressed on/in the 
cellular components of the innate immune system. Toll-like receptors (TLRs) are the best characterized PRRs. Receptorligand interaction via TLRs induces the production of antimicrobial peptides and proinflammatory cytokines through NF- $\kappa \mathrm{B}$, mitogen-activated protein kinase (MAPK) and other signaling pathways (Kawai and Akira, 2006). As a result, TLR signaling is critical to development of the host innate immune response, including recruitment of dendritic cells (DCs) and T effector cells, and upregulation of MHC I and II on antigen presenting cells (APCs) and by extension adaptive immunity against infection. 10 TLRs in human and 13 in the mouse have been identified to date (Kawai and Akira, 2006). TLR2, TLR4, TLR5, and TLR9 recognizing lipopeptide, lipopolysaccharides, flagellin and CpG DNA, respectively, are known to be important in controlling bacterial infections. With the exception of TLR3, the TLRs require the adapter molecule myeloid differentiation factor 88 (MyD88) for signal transduction (Kawai and Akira, 2007). As expected, MyD88 have been shown to be essential for clearance of Brucella infection from mice (Weiss et al., 2005; Copin et al., 2007; Macedo et al., 2008).

Several groups have investigated the contribution of TLR signaling to innate immunity against Brucella infection in the mouse model. The consensus opinion is that TLR2 is not required to control Brucella infection in the mouse (Campos et al., 2004; Copin et al., 2007). However, TLR2 has been shown to be important for cytokine production (Huang et al., 2003; Giambartolomei et al., 2004; Weiss et al., 2005; Macedo et al., 2008; Zwerdling et al., 2008), MHC-II expression (Barrionuevo et al., 2008) and down regulation of the type I receptor for the Fc portion of IgG (Fc $\gamma$ RI, CD64) (Barrionuevo et al., 2011) in tissue culture.

The role of TLR4 in Brucella infection remains controversial. Some studies suggest that TLR4 is required to control Brucella replication in the mouse (Campos et al., 2004; Copin et al., 2007; Macedo et al., 2008); others indicate that TLR4 is not involved (Weiss et al., 2005; Barquero-Calvo et al., 2007). The use of different Brucella strains/species in these experiments may account for the observed differences. The influence of the route of infection on the role of TLRs in studies with other pathogens suggests a need to do so with brucellosis, as is the role of TLR-mediated innate immunity in the development of adaptive immune response. These studies employed intraperitoneal (i.p.) inoculation, which is not a typical route for Brucella infection. As a result of these reports, the consensus of scientific opinion is that MyD88 contributes significantly to the control of Brucella infection while TLR-based signaling plays a lesser role at best. The absence of any role for TLR signaling is consistent with results indicating that the Brucella protein TcpB interrupts TLR-based signaling by promoting degradation of MyD88 adaptor like protein, MAL (Chaudhary et al., 2012), and modification of Brucella LPS reduces agonist activity (Duenas et al., 2004). However, these studies have been restricted to an atypical route of exposure, and the potential for differential TLR expression associated with different tissues has not been considered (Juarez et al., 2010). Since inhalation represents a major concern to public health, experiments were undertaken to determine the role of TLR signaling in the control of Brucella infection following aerosol exposure. The current study, investigates the roles of TLR2, TLR4, and
MyD88 in clearance of Brucella following respiratory exposure and development of adaptive immune response against Brucella. Since the mucosal/respiratory system is the primary portal of entry for human infection documented in many laboratory incidents and relevant to biothreats, it is important to understand the pathogenesis and immune responses resulting from aerosol exposure.

Since TLR signaling is an important component bridging innate and adaptive immunity (Iwasaki and Medzhitov, 2004), failure to clear Brucella from organs by TLR signaling deficient mice could be due to defects in the development of adaptive immunity. To test this hypothesis, we determined cellular- and humoral-mediated adaptive immunity in these TLR knockout mice following Brucella infection. The results reveal that TLR signaling exhibits significant differences in control and clearance of Brucella from selected tissues and in the associated development of an adaptive immune response.

\section{MATERIALS AND METHODS BACTERIAL STRAIN AND GROWTH CONDITIONS}

The bacterial strain used in these experiments, B. melitensis $16 \mathrm{M}$ was obtained from ATCC (\#23456) and recovered from an aborted goat fetus. Bacterial cultures were prepared as previously described (Pei and Ficht, 2004). Briefly, B. melitensis $16 \mathrm{M}$ was cultured in TSB for $24 \mathrm{~h}$, pelleted by centrifugation at 20,000 $\times \mathrm{g}$, washed with and resuspended in PBS ( $\mathrm{pH}$ 7.4) at a density of approximately $5 \times 10^{9} \mathrm{CFU} / \mathrm{ml}$ (Kahl-McDonagh et al., 2007).

\section{MOUSE MODEL OF INFECTION}

Breeding pairs of TLR2 $2^{-/-}$, TLR $4^{-/-}$, and MyD88 $8^{-/-}$mice were obtained from Dr. S. Akira (Osaka University, Osaka, Japan) via Dr. Michael Berton (University of Texas at San Antonio, San Antonio, TX) and colonies maintained by Comparative Medicine Program (CMP) personnel at TAMU. C57BL/6 control mice were purchased from Jackson Laboratory. All mice were housed in BSL-3 suite in the CMP at Texas A\&M University. Mice of both sexes between 8 and 12 weeks old were used in the experiments. Euthanasia was performed using carbon dioxide inhalation. All personnel working with animals received training in rodent handling and euthanasia via the CMP. All animal work was performed in compliance with the Public Health Service (PHS) Policy on Humane Care and Use of Laboratory Animals as described by the National Research Council's Institute for Laboratory Animal Research (ILAR) Guide for the Care and Use of Laboratory Animals.

\section{AEROSOL EXPOSURE OF MICE}

Aerosols were generated via nebulization of a Brucella suspension $\left(\sim 5 \times 10^{9} \mathrm{CFU} / \mathrm{ml}\right.$ in PBS) into a Madison Chamber (Madison, Wisconsin) according to the manufacturer's instructions (KahlMcDonagh et al., 2007). The aerosol chamber was located in a biosafety level 3 (BSL3) facility in the Laboratory Animal Research and Resources (LARR) building staffed and managed by the CMP at Texas A\&M University. To assure personal safety, the principal investigator implemented a strict safety protocol, and all procedures were approved by the IBC and IACUC. Following aerosol exposure, one group of mice $(n=5)$ were immediately 
euthanized via carbon dioxide inhalation and the lungs collected to determine the infecting dose (Kahl-McDonagh et al., 2007). At 1, 2, 4, 6, 8, and 10 weeks after exposure, mice were euthanized and lung, spleen and liver were collected and homogenized in $1 \mathrm{ml}$ each of sterile water containing $0.5 \%(\mathrm{v} / \mathrm{v})$ Tween-20. One hundred microliters of appropriate dilution of the homogenized tissue were plated on Farrell's selective medium plates followed by incubation at $37^{\circ} \mathrm{C}$ for at least 3 days. The experimental limit of detection was determined to be $10 \mathrm{CFU}$ per lung, spleen or gram of live, and the results described represent the accumulated data from 4 independent aerosol exposures.

\section{LYMPHOCYTE PROLIFERATION ASSAY}

At 8-week post infection (p.i.), one-half of the spleen from each euthanized mouse was used to produce single cell suspensions as previously described. The cell density was adjusted to $2 \times 10^{6}$ per $\mathrm{ml}$ using complete RPMI-1640 containing 10\% (v/v) heatinactivated FBS, $1 \mathrm{mM}$ non-essential amino acid, $100 \mu \mathrm{g} / \mathrm{ml}$ of penicillin and $100 \mathrm{U} / \mathrm{ml}$ streptomycin. The cell suspension was dispensed to 24-well plates with $1 \mathrm{ml} /$ well and stimulated with heat killed Brucella (HKB) or ConA $(2 \mu \mathrm{g} / \mathrm{ml})$ for three days. Following stimulation, supernatants were collected and cytokine synthesis characterized. Cells collected from $100 \mu \mathrm{l}$ of the suspension were lysed using 1\% (v/v) Triton X-100, and the LDH released from live cells was determined using CytoTox $96^{\mathrm{TM}}$ Nonradioactive Cytotoxicity Assay kit (Promega) following the manufacturer's instructions.

\section{CYTOKINE ELISA}

IFN- $\gamma$ levels in the culture supernatants were determined $72 \mathrm{~h}$ post stimulation using sandwich ELISA kits (PeproTech Inc., Rocky Hill, NJ) according to the manufacturer's instructions (Pei et al., 2008).

\section{ANTI-BRUCELLA ANTIBODY DETECTION}

Blood samples were collected just prior to euthanization from mice at 1, 2, 4, 6, 8, and 10 weeks post exposure. Sera were separated and anti-Brucella antibodies, including $\operatorname{IgG}, \operatorname{IgG}_{1}, \operatorname{IgG}_{2 \mathrm{a}}$, IgM, and IgA were evaluated using ELISA. ELISA plates (Nunc) were coated with $B$. melitensis $16 \mathrm{M}$ cell lysate. Briefly, $100 \mu \mathrm{l}$ of $1 \mu \mathrm{g} / \mathrm{ml}$ lysate in bicarbonate/carbonate buffer $\left(2.93 \mathrm{~g} \mathrm{NaHCO}_{3}\right.$, $1.59 \mathrm{~g} \mathrm{Na}_{2} \mathrm{CO}_{3}, 0.203 \mathrm{~g} \mathrm{MgCl}_{2}$ in $1 \mathrm{~L}$ of distilled water, $\mathrm{pH} 9.6$ ) was added to microtiter wells (96 wells/plate) and incubated overnight at $4^{\circ} \mathrm{C}$ (Pei and Collisson, 2005). Non-specific binding was blocked via incubation with $5 \%(\mathrm{w} / \mathrm{v})$ non-fat milk in PBS for $1 \mathrm{~h}$ at room temperature. The antigen-coated wells were incubated $2 \mathrm{~h}$ at room temperature $\left(25^{\circ} \mathrm{C}\right)$ with mouse sera diluted in PBS (pH 7.4) containing 0.05\% (v/v) Tween-20 (PBS-T) 200 times for IgG1, IgG 2 , IgM, IgA detection or 1000 times for IgG detection. The plates were then incubated with HRP-conjugated goat anti-mouse immunoglobulins ( $\operatorname{IgG}_{1}, \operatorname{IgG}_{2 \mathrm{a}}$, IgM, IgA, and IgG from Kirkegard-Perry Labs (KPL) for $1 \mathrm{~h}$ at room temperature. The wells were washed with PBS-T between steps to remove any unbound materials. Color development followed the addition of $100 \mu \mathrm{l}$ of TMB substrate KPL. The reaction was terminated by the addition of $50 \mu \mathrm{l}$ of stop buffer $\left(1 \mathrm{M} \mathrm{H}_{2} \mathrm{SO}_{4}\right)$ and $\mathrm{OD}_{450}$ was determined using an ELISA reader (Bio-Rad).

\section{STATISTICAL ANALYSIS}

Statistical significance was determined using one-way ANOVA or Student's $t$-test. $P$-values $<0.05^{*}$ and $<0.01^{* *}$ were considered to be significant.

\section{RESULTS}

\section{THE ROLE OF TLR SIGNALING IN CLEARANCE OF BRUCELLA} FROM THE LUNG

In these experiments, the infecting dose determined using lungs collected from control C57BL/6 mice $(n=5)$ immediately following challenge is $4.26 \pm 0.28 \mathrm{log} \mathrm{CFU} /$ mouse, which is consistent with a previous report (Kahl-McDonagh et al., 2007). Bacterial load recovered from the lungs of TLR2 ${ }^{-/-}$, TLR4 ${ }^{-/-}$, and $\mathrm{MyD} 88^{-/-}$mice during the first two weeks following exposure is not significantly different from the C57BL/6 control mice (Figure 1, panels W1-2), indicating that TLR2, TLR4, and MyD88 are not required to clear lung infection early. A significant difference in bacterial burden is detectable by week 4 and extends through week 10, indicating that the contribution of TLR2, TLR4, and MyD88 to the control of Brucella infection in the lungs occurs via adaptive immunity (Figure 1). By week 4 p.i., there was a significant difference in the clearance of the organism from each of the knockouts. This pattern persisted through week 10, although significance is lost due to the reduction in the number of animals available at this time point. The absence of MyD88 appears to have a greater impact on the clearance of infection from the lungs over this period, as the bacterial burden in the lungs of MyD $88^{-/-}$mice is significantly higher than that in TLR2 ${ }^{-/-}$and TLR $4^{-/}$mice by week 8 p.i. (Figure 1, panel W8). The kinetics of Brucella clearance from the lungs is summarized in Figure 1K, and taken together, these results indicate that TLR2, TLR4, and MyD88 contribute to Brucella clearance from the lungs following aerosol challenge.

\section{THE ROLE OF TLR SIGNALING IN BRUCELLA CLEARANCE FROM THE SPLEEN}

Infection and the kinetics of clearance of Brucella from the spleens of mice is notably different from that observed in the lung. Initially, the organism exhibits a delay in systemic distribution to the spleen that requires up to 4 weeks to achieve maximum burden. The delay in systemic spread of infection is evident in the spleen and less dramatically in the liver (see below), with bacterial burden in 9 of 13 spleens from TLR $4^{-/-}$and 10 of 13 from $\mathrm{MyD} 88^{-/-}$mice below the detection limit (10 cfu/spleen). This compares with a delay in only 1 of 17 TLR2 ${ }^{-/}-$mice and 5 of $20 \mathrm{C} 57 \mathrm{BL} / 6$ mice. The MyD88 ${ }^{-/-}$knockout appears to have a reduced ability to control the infection as evidenced by the significant difference in spleen colonization by week 4 (Figure 2, panel W4). Once established infection proceeds similarly, although the MyD88-/- and TLR4 ${ }^{-/-}$mice exhibit a delay in clearance relative to the TLR2 ${ }^{-/}$and C57BL6 mice (Figure 2, panels W6-10). Systemic spread and the clearance of infection from the spleen is unaffected by the loss of TLR2, with no significant difference observed between the TLR2 ${ }^{-/-/-}$and wild-type mice over the course of infection (Figure 2, panels W1-10). In contrast, both TLR4 $4^{-/-}$and MyD $88^{-/-}$mice exhibited early delays in systemic spread of infection during the first 


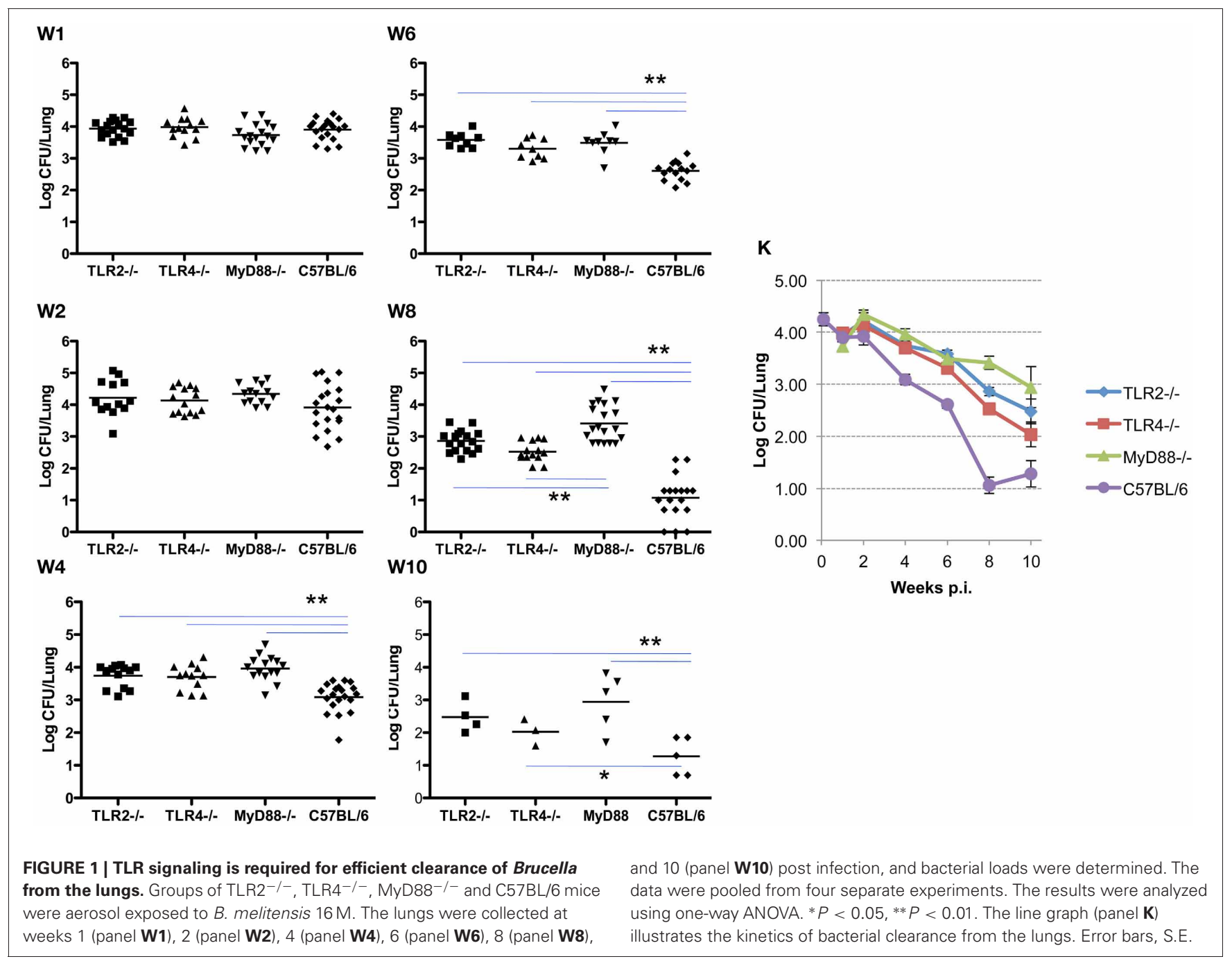

week, which is accompanied by a delay in clearance at later times. Again, the exaggerated decline observed between weeks 8 and 10 in TLR $4^{-/}$mice may be an artifact of the reduced animals numbers.

\section{THE ROLE OF TLR SIGNALING IN BRUCELLA CLEARANCE FROM THE LIVER}

Similar to the results described for the spleen, systemic spread of the organism to the liver is delayed in TLR4 ${ }^{-/-}$and MyD88 $8^{-/-}$ mice, and is associated with a corresponding delay in the clearance of infection. Here again, TLR $2^{-/-}$mice are indistinguishable from control mice in the ability to restrict systemic infection (Figures $\mathbf{2}$ and $\mathbf{3}$ ). As observed in the spleen, the capacity to control infection is most dramatically affected in the MyD88 $8^{-/-}$mice (Figure 3, panels W1-10). From week 2 and onward, TLR2-/and TLR $4^{-/-}$mice controlled infection and cleared the organisms from the livers indistinguishably from the C57BL/6 mice (Figure 3, panel W10). Taken together, these results reveal that, in the mouse liver; (1) TLR2 and TLR4 are not required to control infection or clearance; (2) the absence of TLR4 reduces accumulation of Brucella in the liver, but has little effect on late stage clearance; (3) MyD88 is critical in early control and late clearance of the organism.

\section{ADAPTIVE CELL-MEDIATED IMMUNE RESPONSE FOLLOWING AEROSOL CHALLENGE WITH BRUCELLA}

To determine the development of cell-mediated immunity, the splenocytes collected at 8 weeks p.i. from knockout and control mice are stimulated with Brucella antigen ex vivo. Using an $\mathrm{LDH}$ release assay to measure lymphocyte proliferation, no significant differences in proliferation was detected for TLR2 ${ }^{-/-}, \mathrm{TLR}^{-/-}$, MyD88 $-/-$ and control C57BL/6 mice (Figure 4A). This result indicates that cell-mediated adaptive immunity against Brucella is unimpaired in these knockout mice. Consistent with the results of the lymphocyte proliferation assay, IFN- $\gamma$ levels detected in splenocyte supernatants collected from the knockout mice are not significantly different from C57BL/6 mice (Figure 4B).

\section{ADAPTIVE HUMORAL RESPONSE FOLLOWING AEROSOL CHALLENGE WITH BRUCELLA}

To determine the role of TLR signaling in antibody production and isotype switching, anti-Brucella $\operatorname{IgM}, \operatorname{IgG}, \operatorname{IgG}_{1}, \operatorname{IgG}_{2 \mathrm{a}}$, and 


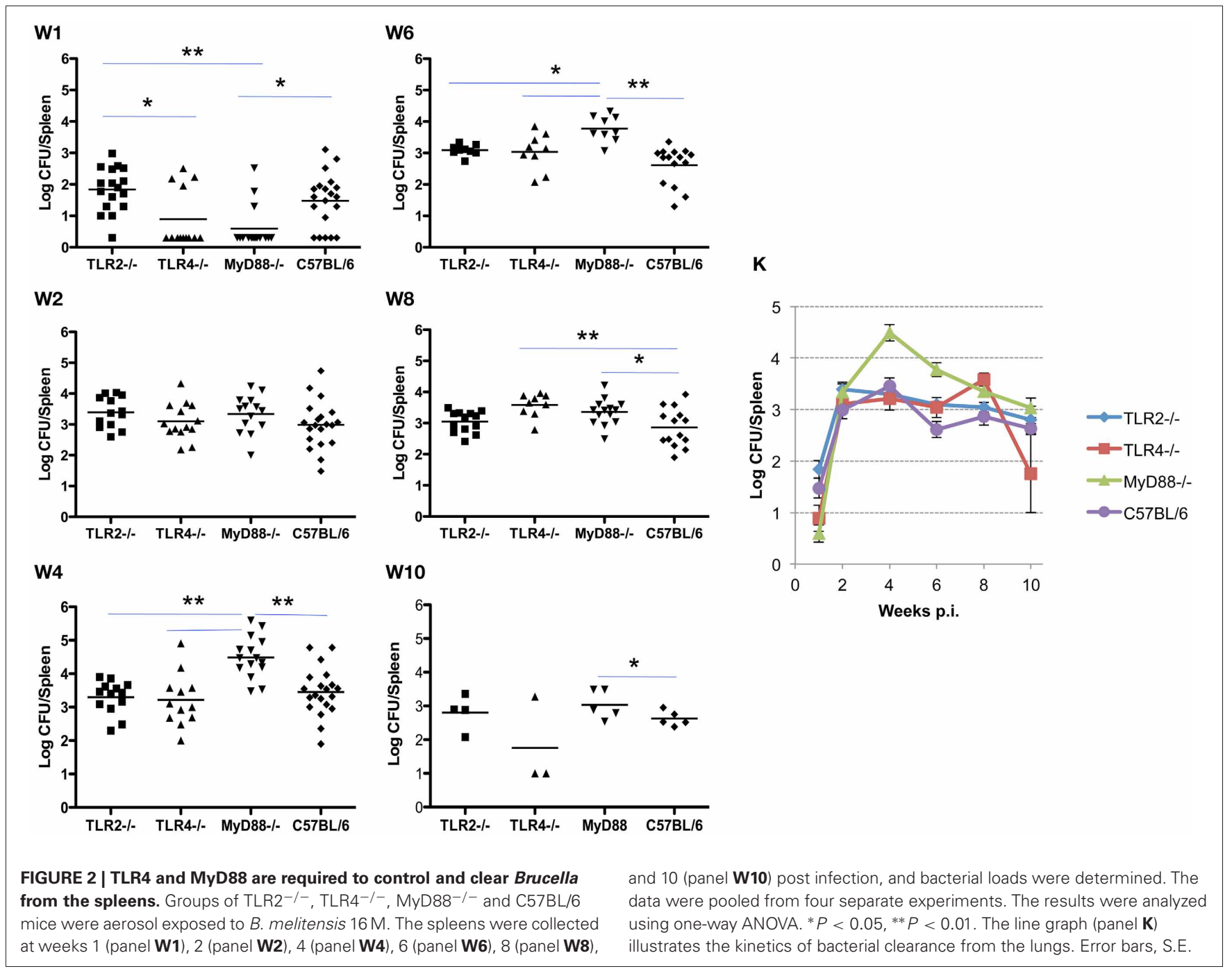

IgA responses were determined following aerosol infection. The results presented reveal detectable levels of IgM by 2 weeks p.i. that peaks by 4 weeks p.i. (Figure 5, panel A). IgM levels begin to decline in the MyD88 $8^{-/}$and C57BL/6 mice at 4 weeks p.i. while, IgM levels in the TLR2 ${ }^{-/-}$and TLR $4^{-/-}$mice plateau at 4 weeks with a gradual decline by 10 weeks p.i. Any requirement for TLR2 and TLR4 in IgM production is transient (only at 4 weeks p.i.). and IgM levels were never significantly different between MyD88 $-/-$ and C57BL/6 mice throughout the experiment.

Total Brucella-specific IgG in wild-type mice is detectable starting 4 weeks post-infection (Figure 5, panel B), and increases continuously over the next 6 weeks. In contrast, elevation of the Brucella specific IgG levels is delayed in all of the knockout mice. Comparison among the groups revealed that, between weeks 4 and 8 p.i., IgG levels in TLR2 ${ }^{-/-}$and TLR $4^{-/-}$mice are significantly lower than that observed in the C57BL/6 mice. IgG levels increase in TLR4 ${ }^{-/-}$and TLR2 ${ }^{-/-}$mice by 10 weeks p.i. to levels that are not significantly different from the control C57BL/6 mice. In contrast, IgG levels in MyD88-/- mice exhibit a significant reduction in titer over the course of infection. Serum IgA is not detected in the TLR2 ${ }^{-/-}$and TLR $4^{-/-}$mice, nor in C57BL/6 mice out to week 10 (Figure 5, panel C). Interestingly, serum IgA is prominent in the sera of $\mathrm{MyD} 88^{-/-}$mice and continuously increases over the course of the experiment.

Recent studies have shown that TLRs play a critical role in determining the fate of naive $\mathrm{T}$ cells, directing them toward either Th1 or Th2 responses (Dabbagh and Lewis, 2003; Xu et al., 2004). To characterize the role of TLR signaling in determining a Th1 or Th2 response, $\operatorname{IgG}_{1}$ (Th2) and $\operatorname{IgG}_{2 \mathrm{a}}$ (Th1) levels and the $\mathrm{IgG}_{1} / \mathrm{IgG}_{2 \mathrm{a}}$ ratio are calculated following Brucella aerosol exposure. $\operatorname{IgG}_{1}$ and $\mathrm{IgG}_{2 \mathrm{a}}$ production are above background levels only 8 weeks p.i. (Figure 5, panels D and E). By week 10, TLR2 and C57BL/6 mice produce significantly elevated levels of $\mathrm{IgG}_{1}$ (Figure 5, panel D) indicating TLR2 is not required for $\mathrm{IgG}_{1}$ production. In contrast, $\mathrm{IgG}_{1}$ levels in TLR4 and MyD88 knockout mice are significantly reduced, indicating TLR4 and MyD88 are essential for $\operatorname{IgG}_{1}$ production (Figure 5, panel D). Relatively low levels of $\operatorname{IgG}_{2 \mathrm{a}}$ are present at 10 weeks p.i. in all groups, but $\mathrm{IgG}_{2 \mathrm{a}}$ levels in TLR2 and MyD88 knockout mice are significantly reduced relative to control mice. $\operatorname{IgG}_{1} / \operatorname{IgG}_{2 a}$ 

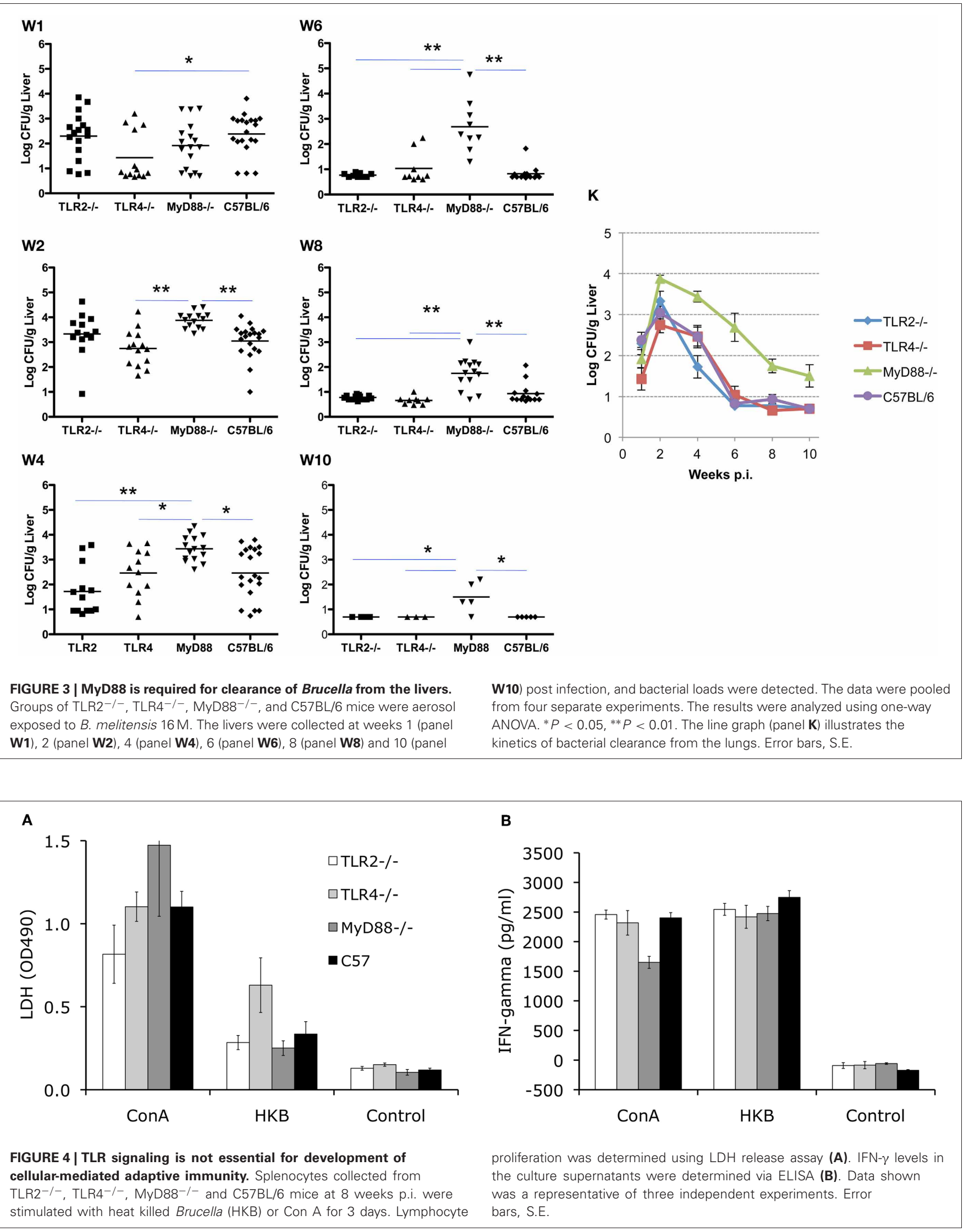


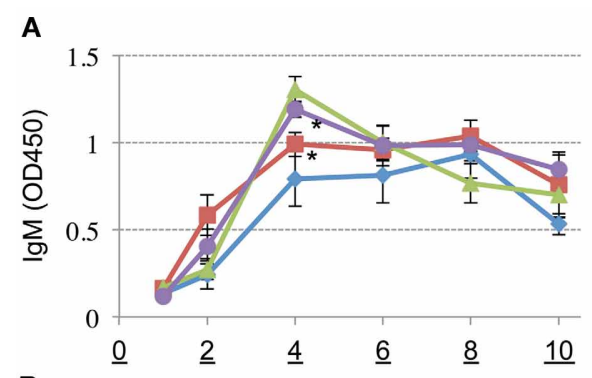

B
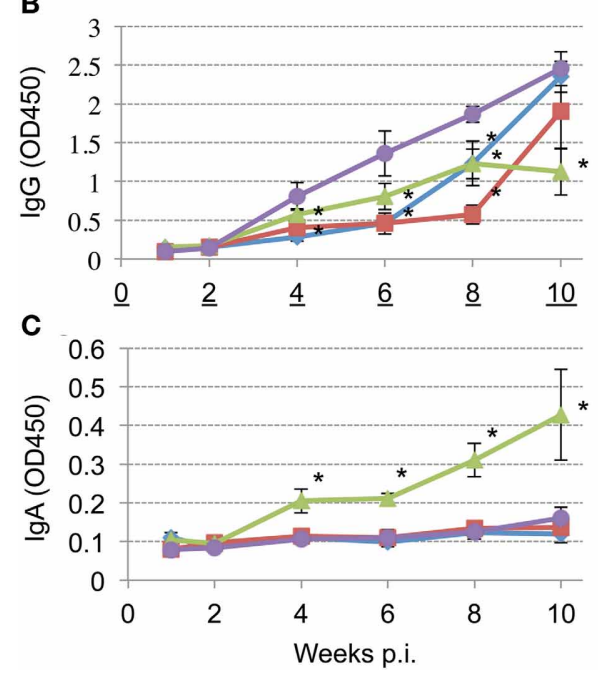

FIGURE 5 | Different TLRs play different roles in eliciting antibody responses after aerosol challenge. Groups of $\mathrm{TLR}^{-/-}, \mathrm{TLR}^{-/-}$, MyD88 ${ }^{-1-}$ and $\mathrm{C} 57 \mathrm{BL} / 6$ mice were aerosol exposed to B. melitensis $16 \mathrm{M}$. Blood samples were collected at 1, 2, 4, 6, 8, and 10 weeks post infection
D
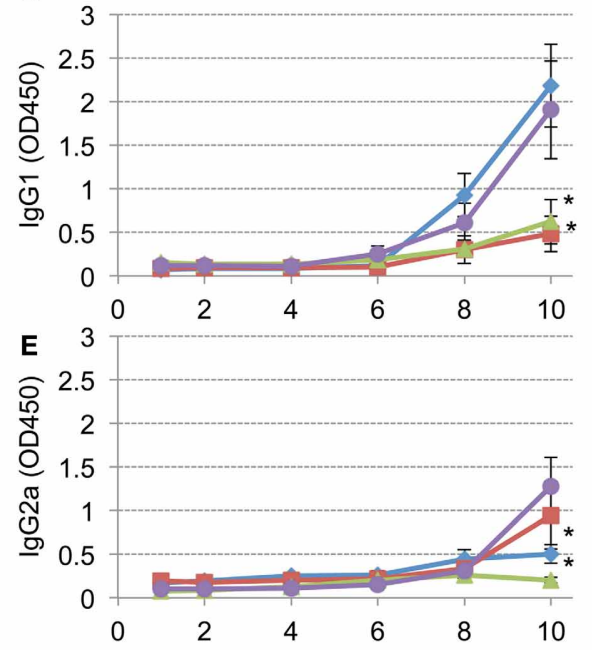

$\mathbf{F}$

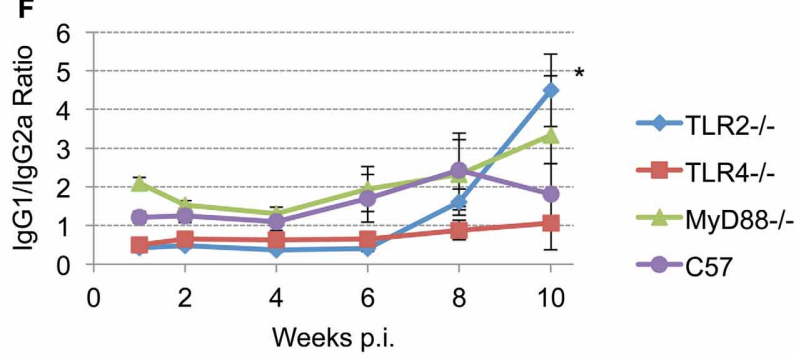

and sera were isolated. Immunoglobulin levels IgM (panel A), IgG (panel B), $\lg A$ (panel C), IgG1 (panel D), IgG2a (panel E) in the sera were detected using ELISA. IgG1/lgG2a ratio is illustrated (panel F). Error bars, S.E. $* P<0.05$ when compared to C57BL/6. ratios suggesting Th2-skewed immune responses are induced in TLR2 ${ }^{-/}-$, MyD88 $8^{-/-}$and C57BL/6 mice. The Th2 response in TLR2 knockout mice is significantly elevated compared to wild type mice at 10 weeks p.i. (Figure 5, panel F), indicating TLR2 interactions suppress Th2 response following aerosol infection.

\section{DISCUSSION}

The kinetics of Brucella spp. clearance from infected mice differs depending on the genetic background of the strain. In C57BL/6 mice, infection is at or below the limit of detection in the liver as early as 6 weeks p.i. or by 8 weeks in the lung (Figures 1 and 3 ). In contrast, these same organs in $\mathrm{BALB} / \mathrm{c}$ mice contain persistent levels of organism 8 weeks p.i. and beyond (Kahl-McDonagh et al., 2007). These results are consistent with previous reports describing enhanced sensitivity of BALB/c mice to Brucella infection following i.p. inoculation (Copin et al., 2007), confirming the expected immunological outcome despite the use of different routes of inoculation. One explanation for the improved clearance from C57BL6 mice is generation of a TH1-skewed immune response as opposed to a $\mathrm{TH} 2$-skewed response in $\mathrm{BALB} / \mathrm{c}$ mice (Watanabe et al., 2004).

TLR activation induces the maturation of APCs, enhances antigen presentation, up-regulation of co-stimulatory molecules and cytokine production. Cytokine profiles produced by APCs control $\mathrm{CD}^{+}{ }^{+} \mathrm{T}$ cell differentiation into either $\mathrm{TH} 1$ or $\mathrm{TH} 2$ cells. Engagement of different TLRs is expected to affect cytokine production, with significant effect on $\mathrm{CD} 4^{+} \mathrm{T}$ cell differentiation (Dabbagh and Lewis, 2003). Previous studies have shown that Brucella infection induces mainly a TH1 response during acute disease (Agranovich et al., 1999; Pasquali et al., 2001; Giambartolomei et al., 2002; Rafiei et al., 2006; Khatun et al., 2009).

It is clear that TLR signaling plays a critical role in the activation of the host innate immune response, including cytokine and chemokine secretion and up-regulation of co-stimulatory molecules in APCs (Kawai and Akira, 2006). Recent studies have shown that these activations indirectly affect the development of adaptive immunity (Iwasaki and Medzhitov, 2010). In addition, it has been demonstrated that functional TLRs are expressed on various T and B cell subsets (Bekeredjian-Ding and Jego, 2009; Booth et al., 2011; Kulkarni et al., 2011). For example, functional expression of TLRs has been extensively investigated on $\gamma \delta$-T cells (Wesch et al., 2011) that have been shown to be important in controlling Brucella infection (Bertotto et al., 1993; Ottones et al., 2000). Interaction of TLRs with their respective microbial ligands provides a third signal for B cell activation, which is essential for optimal antigen-specific antibody production and class switch 
recombination (Bekeredjian-Ding and Jego, 2009; Booth et al., 2011).

Brucella are adept at inhibiting the host immune response. Brucella virulence derives from expression of an LPS with reduced agonist activity that limits activation of innate immunity and development of an effective adaptive response that promotes invasion and establishment of a replicative niche. In addition, Brucella express a protein TcpB (or Btp1) that restricts the proinflammatory response by interfering with MyD88 function. The result is enhanced degradation of MAL (MyD88-adapter-like protein) and restricted expression of NFKB. However, our current results indicate infection varies in different tissues, and that this variation is attributable in part to various PRRs, including TLR2, TLR4, and downstream signaling partners, like MyD88. Interference with TLR signaling by the organism has been described in numerous studies, as has the prominent contribution of MyD88 in limiting infection. The results reported here confirm the importance of MyD88 in the control of infection and extend those findings to include three tissues; spleen, liver and lung. In addition, the experiments performed confirm previous findings concerning the lack of significant contribution of TLR4 and TLR2 with regard to spleen (and liver) infection, but reveal their significant contribution preventing lung infection.

In an effort to explain the control exerted by each of these host factors, an evaluation was made of the corresponding immune response. Since cellular immunity is critical in controlling Brucella infection, splenocytes were used to characterize the role of TLR2, TLR4 and MyD88 in the development of cellmediated adaptive immunity against Brucella. Finding that the loss of TLR2, TLR4 and MyD88 had no demonstrable effect on the development of memory $\mathrm{T}$ cell response against Brucella infection was initially a surprise (Figure 4). However, recent experiments have revealed a disruption of TLR signaling resulting from enhanced degradation of MyD88-adaptor like protein (MAL) that is consistent with previous reports documenting a minimal role for TLR signaling in memory $\mathrm{T}$ cell development against other viral and bacterial infections (Way et al., 2003; Fremond et al., 2004; Heer et al., 2007; Seibert et al., 2010; McBride et al., 2011). These results are also in agreement with a recent study showing that IRAK4 (interleukin-1 receptor associated kinase 4), one of the kinases recruited by MyD88 upon stimulation, is not required for generation of $\mathrm{CD} 4+$ and $\mathrm{CD} 8+$ $\mathrm{T}$ cells producing IFN- $\gamma$ in the late stage of Brucella infection (Oliveira et al., 2011). These results suggest that TLR-independent signaling is likely involved in the development of cellular adaptive immunity against Brucella.

The contribution of humoral immunity to the outcome of Brucella infection is controversial. IgM may enhance opsonic uptake or the activity of complement. However, the differences in IgM production between knockouts are only transitory and have no correlation with differences in immune clearance. In general, IgG levels are significantly reduced in each of the knockout mice. However, the effect in the TLR4 and TLR2 knockouts is transient, while reduced IgG levels are consistently observed in the absence of MyD88 function. A second marker associated with the loss of MyD88 is the significant level of circulating IgA observed in the MyD88 knockout mice. Surprisingly, serum IgA levels were elevated in ${\mathrm{MyD} 88^{-/-}}^{-}$mice, but not in TLR2 ${ }^{-/-}$and TLR4 $4^{-/-}$ mice following Brucella infection, suggesting that the IgA class switch recombination or secretion is enhanced in MyD88 knockout mice. Alternatively, higher Brucella burden in the lung constantly stimulates IgA secretion. However, this hypothesis is not supported by the fact that serum IgA is not detected in the TLR2 and TLR4 knockout mice despite similar levels of bacterial burden in the lung. In fact, this phenomenon has been previously reported in MyD88 $8^{-/}$mice orally vaccinated with attenuated Salmonella expressing a Streptococcus pneumoniae surface antigen (Park et al., 2008). It could prove useful to understand the biological mechanism involved and its potential application to vaccine design. The contribution of either elevated IgA or suppressed IgG to the inability of MyD88 knockouts to control infection is under investigation.

Since a reduction in the IgG1/IgG2a ratio is associated with the development of a $\mathrm{T}_{\mathrm{H}} 1$ response and protection against Brucella infection, IgG, IgG1 and IgG2a levels were characterized in TLR2, TLR4 and MyD88 following aerosol exposure (Figure 5, panel F). The results reveal a significant contribution of TLR4 and MyD88 to IgG1 production and the importance of TLR2 and MyD88 to IgG2a production. Examination of the IgG1/IgG2a ratio suggests the dependence of a protective $T_{H} 1$ response on expression of MyD88 and TLR2, but not TLR4. This is not a totally unexpected result since the Brucella LPS, and/or TcpB primarily exert their influence on TLR4-based signaling. The contribution of MyD88 and TLR2 to protective immune response as determined by the reduced bacterial burden in selected tissues is borne out in previous discussion. To reiterate, MyD88 ${ }^{-/-}$exhibits significant delays in clearance from all three tissues examined; TLR2 and TLR4 contribute to clearance from the lung alone.

The importance of TLR signaling in the induction of antibody production remains controversial. In one such study, it was shown that TLR is not required for mice to generate robust antibody response (Gavin et al., 2006). Other researchers report that TLR signaling is critical for antibody production and isotype switching (Pasare and Medzhitov, 2005; Heer et al., 2007). A recent study by Weiss et al. show that TLR2, TLR4, TLR2/TLR4, and MyD88 are not required for anti-Brucella IgG production (Weiss et al., 2005). In contrast, our results reveal that production of Brucella specific IgG is delayed in TLR2 and TLR4 knockout mice and impaired in MyD88 knockout mice. Possible reasons for the discrepancies include the use of different Brucella species, B. melitensis $16 \mathrm{M}$ vs $B$. abortus $\mathrm{S} 19$, differences in virulence and different routes of inoculation (aerosol exposure vs. intraperitoneal injection). Since IgM production is not affected by MyD88 deficiency and only transiently affected by TLR2 and TLR4 absence, we conclude that TLR2, TLR4 and MyD88 are critical for IgG class switch recombination, which might be critical to the control of Brucella infection, and should be considered when Brucella designing vaccines.

In our current report, we have clearly demonstrated that TLR2 and TLR4 are critical to clearance of Brucella infection from the lung, although less prominently than MyD88. This contrasts significantly with the clearance profile for the spleen and liver and may arise as a result of differences in the TLR expression pattern or levels reported for different tissues (Nishimura and Naito, 2005). It should be noted that based on results of previous studies 
following i.p. inoculation in which only the spleens were examined, it was concluded that Brucella infection was unaffected by the loss of TLR2, however, bacterial load in the lungs and livers were not determined in these studies. (Campos et al., 2004; Weiss et al., 2005; Copin et al., 2007; Macedo et al., 2008). In agreement with previous studies, the results confirm MyD88 as a critical factor in the control of Brucella infection in the spleen (Campos et al., 2004; Weiss et al., 2005; Barquero-Calvo et al., 2007; Copin et al., 2007; Macedo et al., 2008). However, caution is warranted in interpretation of the significance of host factors based on the analysis of a single tissue. Recent results in our laboratory indicate that Brucella may be recovered from the lung following i.p. inoculation, indicating a need to consider persistence in this tissue irrespective of the route of infection.

Another unexpected observation in this study was the delay in infection of the spleens and livers of TLR4 ${ }^{-/-}$and $\mathrm{MyD} 88^{-/-}$ mice 1-2 weeks post-inoculation. These results suggest that TLR4 and MyD88 play significant roles in mediating Brucella dissemination from the lungs to other tissues, which is consistent with a previous observation indicating a role for TLR4 in the uptake of smooth Brucella by macrophage (Pei et al., 2008), and that translocation of bacteria across mucosal or intestinal barriers is mediated by TLR4 (Neal et al., 2006). Evaluation of Brucella infection dynamics derived from different routes of entry during the first week post aerosol exposure may be expected to confirm these results and potentially suggest post-exposure treatments.

Over millions of year's co-evolution between host and pathogen have resulted in strategies to survive in the host and cause disease presumably without eliminating the host entirely. Recent studies reveal that bacteria exploit the host TLR pathway, resulting either in enhanced virulence or immune suppression (Arpaia et al., 2011; Round et al., 2011). Our previous study has shown that Brucella can use TLR4 to gain entry into the host cells (Pei et al., 2008). It is possible that Brucella inhibit IgA class switch recombination or secretion via MyD88 by an unknown mechanism in order to initiate infection through mucosal surfaces.

Overall, our data demonstrate for the first time that TLR2, TLR4, MyD88 are essential for clearance of Brucella from the lung following aerosol exposure. Although TLR2, TLR4 and MyD88 are not required for the development of cell-mediated adaptive immunity, they play diverse roles in Brucella antigen specific antibody production and antibody class switching. The information obtained from this study will greatly facilitate efforts to understand immunity to Brucella and the rational design of novel vaccines against Brucella infection.

\section{ACKNOWLEDGMENTS}

This work was supported by grants from NIH to Thomas A. Ficht (AI-0048496). The authors wish to thank Dr. Michael Berton (University of Texas at San Antonio, San Antonio, TX) for generously sharing breeding pairs of TLR2 ${ }^{-/-}$, TLR4 ${ }^{-/-}$, and MyD88 $8^{-/}$mice with the permission of Dr. S. Akira (Osaka University, Osaka, Japan). The authors also want to acknowledge the invaluable assistance of Dr. David McMurray and Dr. Christine McFarland in the use of the Madison chamber.

\section{REFERENCES}

Agranovich, I., Scott, D. E., Terle, D., Lee, K., and Golding, B. (1999). Down-regulation of Th2 responses by Brucella abortus, a strong Th1 stimulus, correlates with alterations in the B7.2-CD28 pathway. Infect. Immun. 67, 4418-4426.

Arpaia, N., Godec, J., Lau, L., Sivick, K. E., McLaughlin, L. M., Jones, M. B., Dracheva, T., Peterson, S. N., Monack, D. M., and Barton, G. M. (2011). TLR signaling is required for Salmonella typhimurium Virulence. Cell 144, 675-688.

Atluri, V. L., Xavier, M. N., de Jong, M. F., den Hartigh, A. B., and Tsolis, R. M. (2011). Interactions of the human pathogenic Brucella species with their hosts. Annu. Rev. Microbiol. 65, 523-541.

Baldwin, C. L., and Goenka, R. (2006). Host immune responses to the intracellular bacteria Brucella: does the bacteria instruct the host to facilitate chronic infection? Crit. Rev. Immunol. 26, 407-442.

Baldwin, C. L., and Parent, M. (2002). Fundamentals of host immune response against Brucella abortus: what the mouse model has revealed about control of infection. Vet. Microbiol. 90, 367-382.
Barquero-Calvo, E., Chaves-Olarte, E., Weiss, D. S., Guzman-Verri, C., Chacon-Diaz, C., Rucavado, A., Moriyon, I., and Moreno, E. (2007). Brucella abortus uses a stealthy strategy to avoid activation of the innate immune system during the onset of infection. PLOS ONE 2:e631. doi: 10.1371/journal.pone. 0000631

Barrionuevo, P., Cassataro, J., Delpino, M. V., Zwerdling, A., Pasquevich, K. A., Samartino, C. G., Wallach, J. C., Fossati, C. A., and Giambartolomei, G. H. (2008). Brucella abortus inhibits major histocompatibility complex class II expression and antigen processing through interleukin-6 secretion via toll-like receptor 2. Infect. Immun. 76, 250-262.

Barrionuevo, P., Delpino, M. V., Velásquez, L. N., García Samartino, C., Coria, L. M., Ibañez, A. E., Rodríguez, M. E., Cassataro, J., and Giambartolomei, G. H. (2011). Brucella abortus inhibits IFN[gamma]-induced Fc[gamma]RI expression and Fc[gamma]RIrestricted phagocytosis via toll-like receptor 2 on human monocytes/macrophages. Microbes Infect. $13,239-250$.
Bekeredjian-Ding, I., and Jego, G. (2009). Toll-like receptors - sentries in the B-cell response. Immunology 128, 311-323.

Bertotto, A., Gerli, R., Spinozzi, F., Muscat, C., Scalise, F., Castellucci, G., Sposito, M., Candio, F., and Vaccaro, R. (1993). Lymphocytes bearing the gamma delta $\mathrm{T}$ cell receptor in acute Brucella melitensis infection. Eur. J. Immunol. 23, 1177-1180.

Booth, J., Wilson, H., Jimbo, S., and Mutwiri, G. (2011). Modulation of B cell responses by Toll-like receptors. Cell Tissue Res. 343, 131-140.

Campos, M. A., Rosinha, G. M., Almeida, I. C., Salgueiro, X. S., Jarvis, B. W., Splitter, G. A., Qureshi, N., Bruna-Romero, O., Gazzinelli, R. T., and Oliveira, S. C. (2004). Role of Toll-like receptor 4 in induction of cell-mediated immunity and resistance to Brucella abortus infection in mice. Infect. Immun. 72, 176-186.

Chaudhary, A., Ganguly, K., Cabantous, S., Waldo, G. S., Micheva-Viteva, S. N., Nag, K., Hlavacek, W. S., and Tung, C. S. (2012). The Brucella TIR-like protein $\mathrm{TcpB}$ interacts with the death domain of MyD88. Biochem. Biophys. Res. Commun. 417, 299-304.

Copin, R., De Baetselier, P., Carlier, Y., Letesson, J. J., and Muraille, E. (2007). MyD88-Dependent activation of B220-CD11b+LY-6C+ dendritic cells during Brucella melitensis infection. J. Immunol. 178, 5182-5191.

Dabbagh, K., and Lewis, D. B. (2003). Toll-like receptors and T-helper1/T-helper-2 responses. Curr. Opin. Infect. Dis. 16, 199-204.

Dornand, J., Gross, A., Lafont, V., Liautard, J., Oliaro, J., and Liautard, J. P. (2002). The innate immune response against Brucella in humans. Vet. Microbiol. 90, 383-394.

Duenas, A. I., Orduna, A., Crespo, M. S., and Garcia-Rodriguez, C. (2004). Interaction of endotoxins with Toll-like receptor 4 correlates with their endotoxic potential and may explain the proinflammatory effect of Brucella spp. LPS. Int. Immunol. 16, 1467-1475.

Fremond, C. M., Yeremeev, V., Nicolle, D. M., Jacobs, M., Quesniaux, V. F., and Ryffel, B. (2004). Fatal Mycobacterium tuberculosis infection despite adaptive immune 
response in the absence of MyD88. J. Clin. Invest. 114, 1790-1799.

Gavin, A. L., Hoebe, K., Duong, B., Ota, T., Martin, C., Beutler, B., and Nemazee, D. (2006). Adjuvant-enhanced antibody responses in the absence of toll-like receptor signaling. Science 314, 1936-1938.

Giambartolomei, G. H., Delpino, M. V., Cahanovich, M. E., Wallach, J. C., Baldi, P. C., Velikovsky, C. A., and Fossati, C. A. (2002). Diminished production of $\mathrm{T}$ helper 1 cytokines correlates with $\mathrm{T}$ cell unresponsiveness to Brucella cytoplasmic proteins in chronic human brucellosis. J. Infect. Dis. 186, 252-259.

Giambartolomei, G. H., Zwerdling, A., Cassataro, J., Bruno, L., Fossati, C. A., and Philipp, M. T. (2004). Lipoproteins, not lipopolysaccharide, are the key mediators of the proinflammatory response elicited by heat-killed Brucella abortus. J. Immunol. 173, 4635-4642.

Heer, A. K., Shamshiev, A., Donda, A., Uematsu, S., Akira, S., Kopf, M., and Marsland, B. J. (2007). TLR signaling fine-tunes anti-influenza B cell responses without regulating effector $\mathrm{T}$ cell responses. J. Immunol. 178, 2182-2191.

Huang, L. Y., Aliberti, J., Leifer, C. A., Segal, D. M., Sher, A., Golenbock, D. T., and Golding, B. (2003). Heatkilled Brucella abortus induces TNF and IL-12p40 by distinct MyD88dependent pathways: TNF, unlike IL-12p40 secretion, is Toll-like receptor 2 dependent. J. Immunol. 171, 1441-1446.

Iwasaki, A., and Medzhitov, R. (2004). Toll-like receptor control of the adaptive immune responses. Nat. Immunol. 5, 987-995.

Iwasaki, A., and Medzhitov, R. (2010). Regulation of adaptive immunity by the innate immune system. Science $327,291-295$.

Juarez, E., Nuñez, C., Sada, E., Ellner, J. J., Schwander, S. K., and Torres, M. (2010). Differential expression of Toll-like receptors on human alveolar macrophages and autologous peripheral monocytes. Respir. Res. $11,2$.

Kahl-McDonagh, M. M., ArenasGamboa, A. M., and Ficht, T. A. (2007). Aerosol infection of BALB/c mice with Brucella melitensis and Brucella abortus and protective efficacy against aerosol challenge. Infect. Immun. 75, 4923-4932.

Kawai, T., and Akira, S. (2006). TLR signaling. Cell Death Differ. 13, 816-825.

Kawai, T., and Akira, S. (2007). TLR signaling. Semin. Immunol. 19, 24-32.

Khatun, M. M., Islam, M. A., Baek, B. K., and Lee, S. I. (2009). Characteristics of the immune response during acute brucellosis in Sprague-Dawley rats. J. Infect. Dev. Ctries. 3, 392-397.

Kulkarni, R., Behboudi, S., and Sharif, S. (2011). Insights into the role of Toll-like receptors in modulation of T cell responses. Cell Tissue Res. 343, 141-152.

Macedo, G. C., Magnani, D. M., Carvalho, N. B., Bruna-Romero, O., Gazzinelli, R. T., and Oliveira, S. C. (2008). Central role of MyD88dependent dendritic cell maturation and proinflammatory cytokine production to control Brucella abortus infection. J. Immunol. 180, 1080-1087.

McBride, A., Bhatt, K., and Salgame, P. (2011). Development of a secondary immune response to Mycobacterium tuberculosis is independent of tolllike receptor 2. Infect. Immun. 79, 1118-1123.

Neal, M. D., Leaphart, C., Levy, R., Prince, J., Billiar, T. R., Watkins, S., Li, J., Cetin, S., Ford, H., Schreiber, A., and Hackam, D. J. (2006). Enterocyte TLR4 mediates phagocytosis and translocation of bacteria across the intestinal barrier. $J$. Immunol. 176, 3070-3079.

Nishimura, M., and Naito, S. (2005). Tissue-specific mRNA expression profiles of human toll-like receptors and related genes. Biol. Pharm. Bull. $28,886-892$.

Oliveira, F. S., Carvalho, N. B., Brandao, A. P. M. S., Gomes, M. T. R., de Almeida, L. A., and Oliveira, S. C. (2011). IL-1 receptor-associated kinase 4 (IRAK-4) is essential for initial host control of Brucella abortus infection. Infect. Immun. 79, 4688-4695.

Ottones, F., Liautard, J., Gross, A., Rabenoelina, F., Liautard, J. P., and Favero, J. (2000). Activation of human Vgamma9Vdelta2 $\mathrm{T}$ cells by a Brucella suis non-peptidic fraction impairs bacterial intracellular multiplication in monocytic infected cells. Immunology 100, 252-258.

Park, S. M., Ko, H. J., Shim, D. H., Yang, J. Y., Park, Y. H., Curtiss, R. 3rd., and
Kweon, M. N. (2008). MyD88 signaling is not essential for induction of antigen-specific B cell responses but is indispensable for protection against Streptococcus pneumoniae infection following oral vaccination with attenuated Salmonella expressing PspA antigen. J. Immunol. 181 6447-6455.

Pasare, C., and Medzhitov, R. (2005) Control of B-cell responses by Toll-like receptors. Nature 438 , 364-368.

Pasquali, P., Adone, R., Gasbarre, L. C., Pistoia, C., and Ciuchini, F. (2001). Mouse cytokine profiles associated with Brucella abortus RB51 vaccination or $B$. abortus 2308 infection. Infect. Immun. 69 , 6541-6544.

Pei, J., and Collisson, E. W. (2005) Specific antibody secreting cells from chickens can be detected by three days and memory $B$ cells by three weeks post-infection with the avian respiratory coronavirus. Dev. Comp. Immunol. 29, 153-160.

Pei, J., and Ficht, T. A. (2004). Brucella abortus rough muntants are cytopathic for macrophages in culture. Infect. Immun. 72, 440-450.

Pei, J., Turse, J. E., and Ficht, T. A. (2008). Evidence that Brucella abortus OPS dictating uptake and restricts NF-кB activation in murine macrophages. Microbes Infect. 10, 582-590.

Rafiei, A., Ardestani, S. K., Kariminia, A., Keyhani, A., Mohraz, M., and Amirkhani, A. (2006). Dominant Th1 cytokine production in early onset of human brucellosis followed by switching towards Th2 along prolongation of disease. J. Infect. 53, 315-324.

Round, J. L., Lee, S. M., Li, J., Tran, G., Jabri, B., Chatila, T. A., and Mazmanian, S. K. (2011). The tolllike receptor 2 pathway establishes colonization by a commensal of the human microbiota. Science 332, 974-977.

Seibert, S. A., Mex, P., Köhler, A., Kaufmann, S. H. E., and Mittrücker, H.-W. (2010). TLR2-, TLR4- and Myd88-independent acquired humoral and cellular immunity against Salmonella enterica serovar Typhimurium. Immunol. Lett. 127, 126-134.

Watanabe, H., Numata, K., Ito, T., Takagi, K., and Matsukawa, A. (2004). Innate immune response in Th1- and Th2-dominant mouse strains. Shock 22, 460-466.

Way, S. S., Kollmann, T. R., Hajjar, A. M., and Wilson, C. B. (2003). Cutting edge: protective cellmediated immunity to Listeria monocytogenes in the absence of myeloid differentiation factor 88 . J. Immunol. 171, 533-537.

Weiss, D. S., Takeda, K., Akira, S., Zychlinsky, A., and Moreno, E. (2005). MyD88, but not toll-like receptors 4 and 2, is required for efficient clearance of Brucella abortus. Infect. Immun. 73, 5137-5143.

Wesch, D., Peters, C., Oberg, H.H., Pietschmann, K., and Kabelitz, D. (2011). Modulation of gammaDelta $\mathrm{T}$ cell responses by TLR ligands. Cell. Mol. Life Sci. 68, 2357-2370.

$\mathrm{Xu}, \mathrm{D}$., Liu, H., and Komai-Koma, M. (2004). Direct and indirect role of Toll-like receptors in $\mathrm{T}$ cell mediated immunity. Cell. Mol. Immunol. 1, 239-246.

Zwerdling, A., Delpino, M. V., Barrionuevo, P., Cassataro, J., Pasquevich, K. A., Garcia Samartino, C., Fossati, C. A., and Giambartolomei, G. H. (2008). Brucella lipoproteins mimic dendritic cell maturation induced by Brucella abortus. Microbes Infect. 10, 1346-1354.

Conflict of Interest Statement: The authors declare that the research was conducted in the absence of any commercial or financial relationships that could be construed as a potential conflict of interest.

Received: 20 January 2012; accepted: 15 August 2012; published online: 07 September 2012.

Citation: Pei J, Ding X, Fan Y, RiceFicht A and Ficht TA (2012) Toll-like receptors are critical for clearance of Brucella and play different roles in development of adaptive immunity following aerosol challenge in mice. Front. Cell. Inf. Microbio. 2:115. doi: 10.3389/fcimb. 2012.00115

Copyright (ㅇ 2012 Pei, Ding, Fan, Rice-Ficht and Ficht. This is an openaccess article distributed under the terms of the Creative Commons Attribution License, which permits use, distribution and reproduction in other forums, provided the original authors and source are credited and subject to any copyright notices concerning any third-party graphics etc. 